\title{
Editorial note to: \\ Matvei P. Bronstein, Quantum theory of weak gravitational fields
}

\author{
S. Deser • A. Starobinsky
}

Published online: 8 November 2011

(C) Springer Science+Business Media, LLC 2011

Keywords Quantization of gravity · Bronstein · Golden Oldie

Bronstein's is only the second work, after L. Rosenfeld's 1930 papers [1,2], on the quantization of (linearized) gravity. It was carried out in 1935, at a time when modern quantum field theory was in its infancy-well before Fierz and Pauli's treatment of spin 2, and soon after Dirac's (1931) and Fermi's (1934) formulations of QED. Its birthplace-Leningrad_-was also remarkable: here a small group of young theoreticians were forming the nucleus of the future Landau school and many other eminent Russian institutions. Indeed, Bronstein had close ties, both personal and scientific, with Landau and his circle, and the spirit animating this work clearly reflects these affinities of style, recognizable to readers of the Landau \& Lifshitz series. Had Bronstein's life not come to a tragic end only a few years later, in Stalin's purges, he would certainly have fulfilled his promise as a most valuable contributor to forefront theoretical physics.

The present work contains a, still quite readable and modern, exposition of second quantization of the massless spin-2 field in flat space-time, including even such

The republication of the original paper can be found in this issue following the editorial note and online via doi:10.1007/s10714-011-1285-4.

S. Deser $(\varangle)$

Brandeis University, Waltham, MA 02 454,

MC 452-48, Caltech, Pasadena, CA 91125, USA

e-mail: deser@brandeis.edu

A. Starobinsky

Landau Institute for Theoretical Physics,

Russian Academy of Sciences, Kosygina 2,

119334 Moscow, Russia

e-mail: alstar@landau.ac.ru 
essential details as the proper operator ordering required to avoid zero-point energy. The system is quantized in Lorentz gauge, and shown to have positive energy for each of its transverse-traceless graviton excitations. A large part of the paper is devoted to the development of measurement theory for a quantized gravitational theory- the first attempt in this direction, too, obviously influenced by the then-new Bohr-Rosenfeld analysis of quantum electrodynamics. Though the author generally follows their treatment, he shows that the gravitational field is essentially different due to the appearance of another restriction on the accuracy of measurement of gravitational fields-in the case of large matter densities. [Here he concentrates on the Christoffel symbols, as the first derivative equivalents of the EM fields.] From these delicate and carefully treated physical effects, the author also concludes-most wisely-that it would be hardly possible to quantize full non-linear gravity without deep revision of classical space-time notions-prophetic conclusion that remains very much actual!

Going beyond the free-field analysis, Bronstein further treated gravity-matter interaction, concretely through Fock's novel covariantization of the Dirac field. Here the author first considers energy transfer by quantized gravitational waves and shows by lengthy calculation that, in the correspondence limit of large occupation number, the classical Einstein expression follows (after correcting a coefficient given by Einstein!). This shows that his quantization is not only qualitatively, but also quantitatively correct. Finally, following the, also contemporaneous, Fock-Podolsky derivation of Coulomb's law from QED, he obtains Newton's law from his linearized quantum gravity, carefully analyzing how the correct sign of the gravitational potential-i.e., opposite to that of Coulomb's - appears in this formalism; while this sign flip is already built-in at the classical level, it is a gratifying check of a new quantum model's correctness.

Finally, we note again the elegant and thorough style and notation-not only for its time, but even now; as noted at the beginning, it will appeal, not coincidentally, to anyone who has studied Landau-Lifshitz. In summary, this paper certainly deserves revival, not just historically, but for its intrinsic (and pedagogical) value. When it was published, general relativity had fallen into a dormant phase, from which it only awoke two decades later. At last, three quarters of a century since 1935, we begin the observational search for quantum-gravitational effects in primordial gravitational wave background generated during inflation. Published in German in a journal inaccessible today, this accessible Golden Oldie translation is a welcome (re-)addition to our subject!

Acknowledgment SD acknowledges support from NSF PHY-1064302 and DOE DE-FG02-164 92ER 40701 grants. We thank G. Gorelik and J. Stachel for very useful correspondence.

Translator's remark: A more detailed summary of this work was published at the same time in "Journal of Experimental and Theoretical Physics". [Bronstein M. <P.> Quantization of Gravitational Waves. Zhurn. Eksper. Teor. Fiz. 6, 195-236 (1936)]. Fragments of this large article are included in the symposium "Albert Einstein and Theory of Gravity", Mir, Moscow 1979, pp. 433-445. 


\section{Matvei P. Bronstein: a brief biography}

\section{By S. Deser}

Matvei Petrovich Bronstein was born December 2, 1906, and shot in a Leningrad prison in Stalin's purges on February 18, 1938 (rehabilitated posthumously in 1957). A precocious scientist, he was one of the legendary group that included Landau and Gamow at Leningrad University in 1926. His first publications date from 1925; in his one allotted decade, he covered a wide range of frontier problems in cosmology, astrophysics and application of the then new quantum mechanics. For his $\mathrm{PhD}$ thesis, a degree newly re-instituted in the USSR, he chose the work presented here, which he defended in front of I. Tamm and V. Fock in November1935. In addition to his great scientific output and participation in the surge of Soviet science in those early years, he translated Dirac's "Quantum mechanics", works by Bohr and wrote several children's science books. On August 6, 1937, he was arrested in the Great Purge; he was never seen again, despite his widow's being informed that he was sentenced to ten years' prison. For a detailed biography, see Refs. [3] and [4].

\section{References}

1. Rosenfeld, L.: Ann. Physik 5, 113 (1930)

2. Rosenfeld, L.: Z. Physik 65, 587 (1930)

3. Gorelik, G., Frenkel, V.: Matvei Petrovich Bronstein and Soviet Theoretical Physics in the Thirties. Birkhäuser, Boston (1994)

4. Gorelik, G.: Matvei Bronstein and quantum gravity: 70th anniversary of the unsolved problem. Physics-Uspekhi 48(10), 1039-1053 (2005) 